\title{
Individual variability in behavior and functional networks predicts vulnerability using a predator scent model of PTSD
}

David Dopfel ${ }^{\# 1}$, Pablo D. Perez ${ }^{\# 1}$, Alexander Verbitsky², Hector Bravo-Rivera ${ }^{3}$, Yuncong $\mathrm{Ma}^{1}$, Gregory J. Quirk ${ }^{3}$, Nanyin Zhang*1

1Department of Biomedical Engineering, Pennsylvania State University, University Park, PA, USA

2Department of Engineering Science and Mechanics, Pennsylvania State University, University Park, PA, USA

${ }^{3}$ Departments of Psychiatry and Anatomy \& Neurobiology, University of Puerto Rico School of Medicine, San Juan, Puerto Rico

\#: These authors contributed equally to this work.

\section{${ }^{*}$ Address for correspondence:}

Nanyin Zhang, Ph.D

Professor of Biomedical Engineering and Electrical Engineering

The Huck Institutes of Life Sciences

The Pennsylvania State University

W-341 Millennium Science Complex, University Park, PA 16802, USA

Email: nuz2@psu.edu

\section{Acknowledgments}

The present study was partially supported by National Institute of Neurological Disorders and Stroke Grant R01NS085200 (PI: Nanyin Zhang, PhD) and National Institute of Mental Health Grant R01MH098003 and RF1MH114224 (PI: Nanyin Zhang, PhD) and R37MH058883 (PI: Gregory Quirk).

\section{Conflict of interest: none.}

Key words: Post-traumatic Stress Disorder; Vulnerability; Resilience; Rat; Resting-state fMRI 
Abstract:

Only a minority of individuals who experience traumatic event(s) subsequently develop post-traumatic stress disorder (PTSD). However, whether differences in vulnerability to PTSD result from predisposition or a consequence of trauma exposure remains unclear. A major challenge in differentiating these possibilities is that clinical studies focus on individuals already exposed to traumatic experiences, and do not take into account pre-trauma conditions. Here using the predator scent model of PTSD in rats

37 and a longitudinal design, we measured pre-trauma brain-wide neural circuit functional connectivity (FC), behavioral and corticosterone responses to trauma exposure, and posttrauma anxiety. Individual differences in freezing responses to predator scent exposure correlated with differences in pre-trauma FC in a set of neural circuits, especially in

41 olfactory and stress-related systems, indicating that pre-existing function in these circuits

42 could predispose animals to differential fearful responses to threats. Counterintuitively, rats with the lowest freezing showed more avoidance of the predator scent, a prolonged

44 corticosterone response, and higher anxiety long after exposure. This study provides a comprehensive framework of pre-existing circuit function that determines threat response strategy, which might be directly related to the development of PTSD-like behaviors. 


65

\section{Introduction}

Maladaptive response to trauma may lead to chronic stress disorders like posttraumatic stress disorder (PTSD). While trauma exposure is a feature of PTSD, it is not an unequivocal predictor of the disorder. In fact, only a minority of individuals who experience traumatic event(s) subsequently develop PTSD. However, mechanisms underlying this individual difference in vulnerability remain unclear.

It has been suggested that vulnerability to PTSD may result from individual differences in early life events, preexisting conditions, or responses to trauma exposure ${ }^{1-3}$. However, mixed results have been reported. For instance, reduced hippocampal volume has long been considered a marker of PTSD. Nevertheless, some studies suggest that PTSD-related decreases in hippocampal volume might be a consequence of traumatic events and PTSD development, whereas other studies showed that PTSD patients' twins who did not have a trauma experience also had smaller hippocampal volumes, suggesting that reduced hippocampal volume may pre-dispose individuals to developing PTSD ${ }^{4-7}$. These conflicting results are also reported in studies of cortisol levels and brain activity in relation to PTSD ${ }^{8-10}$, which together contribute to our elusive understanding of individual vulnerability to PTSD.

A major obstacle of investigating risk factors of PTSD is rooted in the difficulty of longitudinally monitoring of PTSD development pre- through post-trauma in humans with well-controlled traumatic events. Studies in humans focus on populations already exposed to a variety of uncontrolled traumatic events ${ }^{11}$. Such barriers can be overcome in experimental animals, for which a longitudinal design with controlled traumatic stressors can be conveniently applied. Among many stress paradigms that have been 
71 used to produce rat models of PTSD, predator scent exposure in an inescapable

72 environment is a commonly used psychological stressor ${ }^{12}$. This method mimics a life-

73 threatening situation that is ecologically relevant and physically innocuous, preventing

74 confounding effects of inflammation or pain due to injury. Recent work with this model

75 has found that PTSD-like behaviors are exhibited following exposure to predator scent ${ }^{13}$.

76 Importantly, Cohen et al. distinguished the responses of mal- vs. well-adapted rats,

77 showing a $25-30 \%$ prevalence of PTSD-like behavior in rats exposed to trauma ${ }^{14}$. This work highlights the potential for this animal model to investigate relevant individual variability in PTSD-like responses to trauma. Missing from previous studies, however, is information about preexisting conditions of animals that might predict their immediate and long-term responses to trauma.

Additionally, little is known about whether and how specific defense responses to a threat correlate with susceptibility to maladaptation. Exposure to a stressor elicits different fear responses depending on the perceived proximity and intensity of the threat. Moderate stressors produce passive freezing, while imminent predation threats elicit a fight or flight response ${ }^{15,16}$. However, whether behavioral responses to predator scent is a useful indicator of subsequent development of PTSD is unknown.

Here we employed a longitudinal approach to studying individual variability of behavioral and functional outcomes of the predator scent animal model of PTSD. Restingstate functional magnetic resonance imaging (rsfMRI) data were collected from awake rodents before trauma exposure to record pre-existing neural circuit function using the awake rat rsfMRI approach established in our lab ${ }^{17-20}$. Awake rat rsfMRI avoids confounding factors from anesthetics and permits correlation with behavior ${ }^{21-23}$. We 
94 found that individual differences in behavior and neural circuit function prior to trauma 95 exposure predicted animals' susceptibility to developing PTSD-like behaviors. This

\section{Results}

The experimental schedule was summarized in Fig. 1a. Rats were first scanned using rsfMRI, then exposed to fox urine, and then given an anxiety test approximately one week after exposure.

Freezing responses to fox urine were highly variable across animals

A total of 87 Long-Evans (LE) rats were exposed to a predator scent stressor in the form of a compressed cotton pad sprayed with fox urine in an inescapable cage for $10 \mathrm{~min}$. Freezing and location within the cage were quantified with behavioral tracking software (ANY-maze, Stoelting Co., Reston, VA). Compared to control animals that were exposed to a clean cotton pad, the group exposed to predator scent showed increased freezing (t-test, $t_{87}=3.660, p=0.00044$, Fig. $1 b$, left), and maintained a significantly greater distance from the cotton pad (t-test, $\mathrm{t}_{87}=3.568, \mathrm{p}=0.00060$, Fig. $1 \mathrm{~b}$, right). These data confirm that predator scent exposed animals displayed a significantly greater stress response compared to controls.

Closer examination of animals' freezing time revealed distinct distributions between groups. Fig. 1c showed the histograms of freezing time in both groups. Control 
animals exhibited a unimodal distribution of freezing, whereas predator scent exposed animals displayed large inter-subject variability with a bimodal distribution (Fig. 1c, right panel) fitted with a mixed Gaussian distribution (bimodal Gaussian function provided the best fit relative to uni-modal and tri-modal Gaussian functions, model fit quality (the model with the lowest AIC is the best model to fit the data): $\Delta_{1}(\mathrm{AIC})=11, \Delta_{2}(\mathrm{AIC})=0, \Delta_{3}(\mathrm{AIC})$ = 6; Akaike weight for each model: $\left.w_{1}=0.0039, w_{2}=0.947, w_{3}=0.0488\right)$. To further investigate this variation in freezing behavior, predator scent exposed animals in the top and bottom tertiles of freezing time were separated into high-freezing and low-freezing subgroups, respectively ( $n=21$ for each subgroup). Fig. $1 d$ plotted freezing time as a function of exposure time in high-freezing, low-freezing and control groups. Interestingly, it was found that freezing differed between high- and low-freezing animals even during the first minute of predator scent exposure (two sample t-test, $p<0.009$ ) and this difference became larger over the time course of exposure. There was no difference between low-freezing rats and controls. There was also no significant difference in mobility among all three groups, as evidenced by the maximum speed during exposure (one-way ANOVA, $F_{(2,62)}=0.86, p=0.43$, Supplement Fig. 1).

\section{Low-, but not high-freezing animals were susceptible to developing PTSD-like behaviors}

The fact that freezing time was almost identical between low-freezing and control animals might suggest that low-freezing animals had a similar stress response to controls. However, the spatial distribution of time spent in the cage during exposure suggested a different interpretation (Fig. 2). Control animals did not show a side preference with a near normal distribution of the time spent close to the center of the cage (Fig. 2a). In contrast, 
139 both high and low freezing animals exhibited a leftward skewed distribution of time spent,

140 farther away from the fox urine. The average distance from the cotton pad was

141 significantly greater in both low- and high-freezing animals compared to controls (One-

142 way $\operatorname{ANOVA}, F_{(2,62)}=10.97, p=0.000050$ and $p=0.031$, respectively), indicating

143 avoidance behavior in exposed animals. This difference was also supported by the

144 normalized freezing time at different distances from the cotton pad, as shown in Fig. 2 b.

145 Similar to the distribution of the total time spent in the cage, control animals did not seem

146 to have any preference for the side of the cage in which they froze, whereas both high-

147 and low-freezing animals tended to freeze farther away from the fox urine sample.

148 Relative to high-freezing animals, low-freezing animals showed even more leftward bias.

149 The normalized freezing time was significantly less in the zone $24 \mathrm{~cm}$ away from the pad

150 (Two-way Repeated ANOVA, FGroup $\times$ Zone $(22,682)=3.00, p=0.0000059 ;$ Tukey-Kramer

151 post-hoc test, low- vs high-freezing at $24 \mathrm{~cm}: \mathrm{p}=0.04$ ), indicating that low-freezing

152 animals were somewhat more avoidant than high-freezing animals. This can also be seen

153 in the heat plots of Fig. 2a. Prior to predator scent exposure during the habituation period,

154 the three groups showed no significant differences in freezing time (One-way ANOVA,

155 F Group $\times$ Zone $(2,62)=0.24, p=0.78$, Supplement Fig. 2) or location distribution (the cage

156 was divided into three even-size zones, Two-way ANOVA, F Group $\times$ Zone $(4,186)=1.28, p=$ 157 0.28).

To assess the long-lasting PTSD-like behaviors in exposed animals, we measured 159 anxiety levels using an elevated-plus maze (EPM) 6 days after predator scent exposure.

160 Resembling the observation of increased avoidance, low-freezing animals exhibited 161 heightened long-lasting anxiety compared to both control ( $t$-test, $t=2.24, p=0.030)$ and 
162 high-freezing animals (t-test, $\mathrm{t}=3.37, \mathrm{p}=0.0017$ ), as indicated by the ratio of

163 open/open+close arm time in EPM tests (Fig. 2c). There was no statistical difference

164 between high-freezing and control animals, indicating that low-, but not high-freezing

165 animals were susceptible to developing PTSD-like behaviors following exposure to

166 predator scent. In addition, freezing was positively correlated with the EPM score in

167 exposed animals $\left(R^{2}=0.21, p=0.0002\right)$, but not controls $\left(R^{2}=0.01, p=0.62\right.$, Fig. $\left.2 d\right)$.

168 These results demonstrate that behavioral responses during trauma exposure can predict

169 long-term maladaptation. Furthermore, in a separate experiment (Supplement Fig. 3) low-

170 freezing animals were also associated with other types of PTSD-like behaviors including

171 more marble burying behavior ( $n=5$ each group, two sample t-test, $t=5.47, p=0.048$ ),

172 less habituation to acoustic startle response $(n=9$ each group, two-sample $t$-test, $t=5.3$,

$173 p=0.050)$ and less pre-pulse inhibition $(n=9$ each group, two-sample $t$-test, $t=5.16, p$

$174=0.037)$ than high-freezing animals 6 days post predator scent exposure. This provides

175 additional evidence that low-freezers were vulnerable to developing PTSD-like behaviors. scent exposure ( $0 \mathrm{~min}$, baseline) as well as $30 \mathrm{~min}, 60 \mathrm{~min}$ and $120 \mathrm{~min}$ after exposure

(Fig. 3, 15 controls including 9 exposed to air and 6 exposed to lemon scent, as well as during predator scent exposure with 12 low-freezing and 12 high-freezing animals (we did not use tertiles given a relatively smaller sample size in this experiment). 

ANOVA, $\left.F_{\text {time }}(3,56)=0.8, p=0.50\right)$. High-freezing animals exhibited a relatively short test, $30 \min$ vs $60 \min : p=0.049 ; 0 \min$ vs $60 \min : p=0.54 ; 60 \min$ vs $120 \min : p=0.93$ ).

The peak amplitude of CORT response at 30 min was comparable between high- and low-freezing animals (Tukey-Kramer test, $p=0.59$ ). However, relative to high-freezing rats, the CORT response in low-freezing animals was prolonged, maintained at a high level at $60 \min$ (Tukey-Kramer test, $30 \min$ vs $60 \min : p=1 ; 0 \min$ vs $60 \min : p=0.0005$ ) and had a delayed return to baseline at 120 min (Tukey-Kramer test, 60 min vs 120 min: $p=0.0098 ; 0$ min vs 120 min: $p=1$ ). In addition, low freezers' CORT level was significantly higher at 60 min than high freezers (Tukey-Kramer test, $p=0.0039$ ). CORT response curve with the baseline CORT level (i.e. $0 \mathrm{~min}$ ) being subtracted from each time point. This quantification method took into account both the amplitude and duration of the hormone response. Low freezers showed significantly higher total CORT response than high freezers $(t=5.29, p=0.031$, Fig. $3 b)$, as well as controls $(t=6.58, p$ $=0.017$, Fig. 3b). Furthermore, the total CORT response was significantly correlated with 203 freezing time across all fox urine exposed animals $(R=0.433, p=0.035, n=24$, Fig. 3c), 204 and was also linked to long-lasting anxiety, evidenced by a significant correlation with the 205 EPM score measured 6 days after exposure across all animals tested $(r=0.36, p=0.019$, $206 n=39$, Fig. 3d). Taken together, these data demonstrate that low-freezing animals 
207 displayed prolonged CORT response to predator scent stress, which was related to long208 term anxiety.

Pre-trauma neural circuit functional connectivity predicted fear responses.

In addition to behavioral tests, rsfMRI was performed before trauma exposure to

212 measure preexisting resting-state functional connectivity (RSFC) in neural circuits across

213 the whole brain. Echoing our behavioral data showing difference in freezing during the

214 very beginning of predator scent exposure, RSFC of multiple widespread neural circuits

215 significantly differed between high- and low-freezing animals (false discovery rate (FDR)

$216=0.01$, Supplement Fig. 4a). These regions/circuits have been implicated in stress

217 responses, processing of emotionally valent stimuli, olfactory function and freezing

218 behavior.

To identify the specific neural circuits correlated with the freezing phenotype, we conducted a data-driven correlational analysis for all neural connections (67 regions

221 covering the whole brain, 2211 connections in total) between their RSFC and the 222 cumulative freezing time across all exposed animals (Fig. 4a, upper triangle). 15 neural 223 circuits exhibited a significant correlation between pre-exposure RSFC and freezing time 224 ( $p<0.05$, FDR corrected, Fig. 4a, lower triangle). Relative to high-freezing animals, six 225 of these connections showed stronger connectivity, whereas 9 showed lower connectivity 226 in low-freezing animals, suggesting that the pre-trauma connectivity within these circuits 227 might have predictive value for the freezing behavior during exposure. Fig. 4b showed 228 three representative RSFC maps, respectively generated with one region in these 15 
229 connections as the seed (i.e. anterior cingulate cortex, AON and parabrachial nucleus).

230 Fig. 5 showed the correlational relationship between pre-trauma RSFC and freezing time

231 for all 15 circuits. The diagram of this freezing-associated network was summarized in

232 Fig. 6.

A closer examination of these 15 circuits revealed a comprehensive network particularly involving the olfactory and stress-related brain areas. Specifically, lowfreezing animals showed stronger pre-trauma connectivity in two olfactory circuits (i.e. AON-amygdala, and olfactory bulb-auditory cortex), two septal circuits (i.e. lateral septum

237 (LS)-bed nucleus of the stria terminalis (BNST), medial septum (MS)-prelimbic cortex $(P L)$ ), and two circuits within the stress system (lateral hypothalamus-cortical amygdala and lateral hypothalamus-amygdala). Stronger connectivity in the two olfactory circuits suggest that odor may exert more influence on the amygdala and cortex in low-freezing animals. Additionally, the LS-BNST projection might underlie stronger predator scentinduced stress response in low-freezing animals. In support of this, both the LS and BNST are activated by predator odor ${ }^{24,25}$, and the BNST is actively involved in sustained fear 244 response to diffuse threat ${ }^{26}$. Stronger functional connectivity within the stress system can 245 also contribute to higher stress response and anxiety in low freezing animals.

247 in low-freezing animals, including two parabrachial nucleus (PBn) circuits (PBn-BNST and 248 PBn-hypothalamus), two nucleus accumbens (NAc) circuits (NAc-amygdala and NAc249 visual cortex), two zona inserta (ZI) circuits (ZI-pituitary gland and ZI-visceral cortex), as 250 well as the circuits of lateral olfactory tract nucleus (nLOT)-anterior cingulate cortex (ACC), 251 postsubiculum-agranular insula (AI), and globus pallidus (GP)-paraventricular (PVN) 
252 hypothalamus. PBn is tightly related to olfactory function and electrophysiological data

253 show that calcitonin gene-related peptide (CGRP) in PBn has inhibitory effects in the

254 BNST ${ }^{27}$. Therefore, weaker connectivity in the PBn-BNST circuit suggests less inhibition

255 in BNST and could contribute to heightened anxiety in low freezing animals. Weaker

256 RSFC between the nLOT and ACC can reduce ACC's control over predator scent-

257 triggered defense reaction in low-freezing animals. In addition, the NAc-amygdala circuit

258 mediates avoidance behaviors ${ }^{28}$, and decreased NAc connectivity was consistent with

259 report of the inverse association between NAc activity and the intensity of emotional 260 stress response ${ }^{24}$, which might also be related to goal-directed motor behavior 29.

261 Furthermore, lower connectivity in ZI circuits (i.e. ZI-pituitary gland and ZI-visceral cortex)

262 may be involved in control of sympathetic visceral reactions and the hypothalamus-

263 pituitary-adrenal (HPA) axis activity ${ }^{30}$. Lastly, the GP-PVN hypothalamus projection,

264 known to involve corticotropin-releasing factor (CRF) neurons, were reported to link stress

265 response to motor output ${ }^{31}$.

Taken together, these data indicate that differences in functional connectivity in

267 neural circuits particularly involving the olfactory and stress-related systems might 268 predispose animals to differential stress responses during predator scent exposure, which 269 can be linked to individual difference in subsequent development of PTSD-like behaviors

270 long after trauma exposure.

272 Fear responses and long-term anxiety in fox-urine exposed animals were not due to the 273 novelty of the fox urine scent. 
To rule out the possibility that differential responses in fox-urine exposed animals

275

276

277

278

279

280

281

282

283

284

285

286

287

288

289

290

291

292

293

294

295

296

resulted from the novelty component of the scent, we repeated the experiment with a

lemon scent being used as the control scent in a separate cohort of animals $(n=24)$.

Lemon exposed animals showed virtually identical freezing (Supplement Fig. $5 a$, left, $n=$

6, two-sample t-test, $t=2.0, p=0.16$ ) and avoidance (distance from the pad, Supplement

Fig. 5a, right, two-sample t-test, $t=1.14, p=0.32$ ) compared to animals exposed to no scent (i.e. air, $n=6$ ), which ruled out the possibility that the novelty nature in the scent affected fear responses during exposure. In contrast, the fox urine exposed group $(\mathrm{n}=$ 12) maintained a greater mean distance from the cotton pad (one way ANOVA, $F_{(2,23)}=$ 11.61, $p=0.0004)$ than both air $(p=0.012)$ and lemon $(p=0.0006)$ exposed animals. In addition, fox urine exposed animals showed a trend of more freezing than nontraumatized animals (left, $p=0.056$, lemon and air groups combined). Furthermore, consistent with the findings in the main cohort of animals, low-freezing animals exhibited stronger stress response during fox urine exposure and higher anxiety 6 days after exposure. Supplement Fig. 5b showed heat maps of the spatial distribution of time spent in the cage for the air-exposed, lemon-exposed, fox urine-exposed low freezing, and fox urine-exposed high freezing groups, respectively. The air and lemon groups showed no evident bias for either side of the cage, whereas the low-freezing group showed a strong bias toward the far end (from the pad) of the cage. High freezing rats also showed a bias toward the far end of the cage, but to a lesser extent. This result was also confirmed in the spatial distribution of the normalized freezing time for each group (supplement Fig. 5c), which displayed virtually no spatial bias in air and lemon groups, but leftward skewed distributions in high and low freezing rats, with an even stronger bias in low-freezing rats. 
297 Supplement Fig. 5d showed the long-term anxiety measured using EPM performed 6 298 days post exposure in all four groups of animals. Low freezing animals showed a 299 significantly lower EPM score (one way ANOVA, $F_{(2,17)}=4.2, p=0.033$ ) compared to high 300 freezing animals $(p=0.026)$. Also consistent with our data in the main cohort of animals, 301 freezing time was positively correlated to the EPM score across all fox-urine exposed 302 animals $(r=0.783, p=0.0026$, Supplement Fig. 5e), but not in non-traumatized animals $303(r=0.213, p=0.506$, Supplement Fig. 5e). Taken together, these results ruled out the 304 possibility that differential short-term and long-term behavioral responses in fox-urine 305 exposed animals was due to the novel nature of the fox urine scent.

Differential fear behavior during exposure and long-term anxiety between low- and highfreezing animals were not caused by pre-exposure imaging.

To rule out the possibility that differential fear response during predator scent exposure and long-term anxiety between low- versus high-freezing animals indeed resulted from predator scent exposure as oppose to other factors such as the acclimation and/or imaging procedures, we also divided control animals into tertiles based on their cumulative freezing time. No differences in avoidance behaviors including distance to the 314 cotton pad (two-sample t-test, $t=1.22, p=0.25$ ), the distribution of time spent in the cage 315 (two-way ANOVA, $F_{2,28}=2.20, p=0.13$, also see Supplement Fig. 6a), the distribution of freezing time in the cage (repeated measures ANOVA, $F_{2,154}=0.34, p=0.98$ ), or long-

317 term anxiety level (EPM score, two-sample $t$-test, $t=0.37, p=0.72$ ) were observed 318 between low- and high-freezing control animals. In addition, unlike predator scent319 exposed animals, no neural connections exhibited correlation between pre-exposure 
RSFC and freezing time, and no connection displayed significant RSFC difference between low- and high-freezing control animals (Supplement Fig. 4b), indicating that these animals showed virtually no difference in neural circuit function.

Furthermore, we repeated the fox urine exposure experiment in a separate cohort of animals that were not previously acclimated or imaged (control, $n=20$, fox urine exposed, $n=35$ ). Similar differential response during exposure was found in these animals, with lower freezers generally exhibiting more avoidance from the fox urine (Supplement Fig. 6b). These data suggest that the inherent difference between low and high freezers was not caused by potential differential responsiveness to acclimation. This result was supported by the data showing that all three groups of animals (i.e. low-freezing, high-freezing and control) in the main cohort were acclimated to a similar degree, reflected by their consistent motion levels during imaging (One-way ANOVA, $F_{(2,62)}=1.23$, $p=0.3$, Supplement Fig.4c). These findings are also consistent with our previous publications demonstrating that acclimation/imaging does not mask, nor does it interact with the effect of predator stressor $21,23,32$. Taken together, these results confirmed that differential fear behaviors and anxiety levels between low- and high-freezing animals were not driven by the acclimation/imaging procedures as all control animals underwent the identical procedures including imaging, except for being exposed to predator scent.

\section{Discussion}

Employing a longitudinal design, we investigated animals' individual variability in stress response and subsequent development of PTSD-like behaviors following predator 
342 scent exposure. We found that animals displayed large differences in freezing behavior

343 during fox urine exposure. Animals expressing less freezing exhibited increased

344 avoidance and tended to develop long-lasting heightened anxiety. Importantly, this

345 difference in behavioral responses was apparent at the beginning of predator scent 346 exposure, suggesting pre-existing vulnerability. This notion was supported by the rsfMRI

347 data showing significant pre-trauma difference in neural circuit connectivity between high348 and low-freezing animals. In addition, low-freezing animals showed a prolonged CORT 349 response to predator scent, which was correlated with freezing and long-lasting EPM 350 anxiety measurement. Taken together, this study provides important evidence supporting 351 the hypothesis that preexisting circuit function determines the defense response strategy 352 during threat and that this behavioral response might be directly related to the 353 development of PTSD. It also highlights the importance of studies that analyze factors 354 both before and after trauma for the study of PTSD.

Freezing time alone might not be a reliable measure of response to threat. In rodent behavior testing, freezing is dominantly used as the measure of threat reaction. Although freezing is a universal fear response, it manifests at intermediate levels of predator threat ${ }^{16}$. Meanwhile, other types of threat-induced responses including fight-orflight, which is elicited during imminent threat ${ }^{15}$, and avoidance can counteract the measure of freezing. For this reason, the use of freezing as the only measure for stress response of an individual animal can be problematic if alternative coping strategies are 
364

365

not considered ${ }^{33}$. Indeed, there is growing evidence suggesting the ambiguity of freezing time as it relates to stress ${ }^{15,34}$.

In line with this concept, our data show that animals with the lowest freezing time are in fact the most stressed subgroup. Their highly biased distribution in the total time and freezing time at different distance to the cotton pad indicate that low-freezing animals might have perceived a higher threat level and used strategies other than freezing (e.g. avoidance), particularly at closer proximity to the pad. This result was corroborated by significantly higher total CORT response during exposure in low-freezing animals, relative to both high-freezing and control animals. This higher perceived threat level is likely a crucial factor in maladaptation to stress response (e.g. higher long-lasting anxiety level), which is in line with the literature investigating the role of threat perception in human PTSD diagnoses ${ }^{35}$. Taken together, these results demonstrate that freezing time alone might not be a reliable measure of reaction to threat. These data also highlight the importance of separating subpopulations, in particular in stress studies, as key information can be lost through the averaging of subpopulations that display bidirectional responses.

It has to be noted that lower freezing behavior in low freezers did not reflect a reduced ability to respond to the predator scent. Low-freezing animals exhibited more locomotor activity, measured by distance travelled during exposure (Supplemental Fig. 1, One-way ANOVA, $F_{(2,62)}=8.43, p=0.001$, high freezers vs low-freezers, $\left.p=0.001\right)$, and more active avoidance from the fox urine. Furthermore, during exposure there was no difference in mobility among low-, high-freezing and control groups, reflected by the maximal speed (Supplement Fig. 1), also suggesting that low freezers were not suppressed in their response to the fox scent. Different responses during exposure were 
387 also not originated from any systematic bias in these measures among animals, because during the habituation period prior to exposure, the three groups showed no difference in freezing time (Supplement Fig. 2) or location distribution. Thus, the difference in freezing to the predator scent likely reflects a difference in the defensive strategy selected.

Behavioral and neuroendocrine reactions to a threat predict susceptibility to long-term maladaptation development has been an unresolved issue, largely due to lack of longitudinal studies that follow up the relationship between behavioral reactions during trauma exposure and

397 the development of psychopathology ${ }^{15}$. Our longitudinal study demonstrates that freezing during trauma exposure predicts long-term maladaptation in animals, supporting that specific behavioral responses to a threat might predict susceptibility to long-term PTSD400 like behaviors.

The relationship between the behavioral reaction during stress and vulnerability to stress maladaptation is corroborated by neuroendocrine measures during and following predator scent stress. We observed that in low-freezing (i.e. vulnerable) animals, the 404 CORT response was prolonged and had a delayed return back to baseline after stress, 405 and this pattern of CORT response has been suggested as a marker of maladaptation to 406 stress in PTSD ${ }^{36,37}$. Correspondingly, in our study, both freezing time and the total CORT 407 response were correlated with the long-term anxiety measure. These data are also 408 consistent with the theory that stress hormone production is an important and protective 

414 chronic pathological states.

part of acute stress response triggering negative feedback on CORT production ${ }^{38,39,41,42}$. In fact, it has been shown that supplementing CORT at the time of trauma ${ }^{44}$, or immediately after trauma ${ }^{45}$ has protective effects against PTSD symptom development 38. Taken together, our data indicate that individual variation in behavioral and hormonal responses to stress can be key predictors of the development of emotion regulation and

Pre-trauma neural circuit function predisposes animals to differential stress response.

\section{i) Connections between the olfactory and stress systems}

Our rsfMRI data suggest that sensory inputs play an important role in the stress response and subsequent development of PTSD-like behaviors. AON has been shown to be activated by variable stressors ${ }^{46}$ including predator scent ${ }^{47}$. The mitral/tufted (MT) cells in the olfactory bulb send their processes to AON ${ }^{48}$, which projects to amygdala ${ }^{49}$. This connectivity suggests that the flow of olfactory information through the AON to the amygdala could drive the stress response to predator scent. Stronger AON-amygdala connectivity in low-freezing animals suggest that the olfactory cue could have a stronger impact on amygdala activity, which would lead to stronger stress response. Conversely, weaker nLOT-ACC connectivity in low-freezing animals indicates less ACC control during predator scent exposure, which could affect the switching between freezing and active defense modes ${ }^{50}$. These data also agree with the report that the olfactory cortex and its connections with the stress system are tightly linked to stress hormone responses to predator scent in rodents ${ }^{51}$. Taken together, these results highlight the importance of 
431 sensory input in the stress response during predator scent exposure, which also suggest

432 that weaker sensory drive in the stress system may help promote resilience to trauma.

In addition to stronger sensory-stress connectivity, stronger functional connectivity

434 within the stress system was observed in low-freezing animals, consistent with their

435 higher stress response and anxiety. In particular, it is known that threat-induced autonomic sympathetic responses are mediated by amygdala projections to the lateral hypothalamus ${ }^{15}$. Stronger connectivity in this circuit could result in higher arousal, higher cardiovascular activity and increased muscle tone during predator odor exposure in lowfreezing animals ${ }^{52}$.

ii) BNST circuits

Our data also suggest that BNST may be involved in the neural network associated with differential stress response during predator scent exposure. BNST, as a part of the extended amygdala, is well known to be involved in sustained anxiety when a threat is diffuse and/or uncertain 26,53 such as predator odor ${ }^{25}$. As a result, this region is particularly associated with anxiety ${ }^{54-56}$. The anatomical connections between BNST and LS and 446 PBn have been well documented $27,57,58$, but the specific function of these projections is 447 less well studied. It has been shown that CGRP in the PBn inhibits neurons of the BNST 44827 , and BNST projections to the PBn dampen anxiety response ${ }^{59}$. These data support the 449 idea that a weaker PBn-BNST connection in low-freezing (i.e. vulnerable) animals caused 450 disinhibition of BNST activity, which led to a stronger stress response and heightened 451 long-lasting anxiety. Conversely, LS mediated activity, possibly again driven by olfactory 452 inputs ${ }^{24}$, can potentiate BNST activity in these animals, and generate stronger stress 453 responses and heightened anxiety. 
454

iii) The hypothalamus-GP circuit

455

456

457 to the external GP ${ }^{31}$, which expresses high levels of the primary CRF receptor (i.e.

458 CRFR1). These connections makes GP an entry point connecting the stress system with

459 the basal ganglia, which links the stress-relevant information to the directed movement ${ }^{31}$.

460 Consistent with this notion, our data suggest that the pre-trauma connectivity in this circuit

461 is directly associated with stress-related behaviors in animals.

462

464 freezing animals, given that PL promotes fear expression and avoidance ${ }^{60,61}$. Also 465

473 reticular nucleus (RT) was observed in low-freezing rats. RT is the hub connecting 474 hippocampus with PFC, and reduced connectivity in vulnerable rats might be due to less 475 prefrontal control of hippocampus ${ }^{65}$. Although premature, these results suggest that 
476 preexisting neural circuit differences in vulnerable animals might reflect a sophisticated

477 network, and PTSD should be viewed as a network phenomenon. related to anxiety disorders in humans ${ }^{67}$, although the present study goes beyond static 484 neuroanatomy of individual brain regions and focuses on pre-existing neural circuit 485 function. Furthermore, human data also suggest that a dysregulated CORT response is 486 a predictor of vulnerability to subsequent development of PTSD $41,42,45,68$. Taken together, 487 these results provide compelling evidence supporting the relevance of the present study 488 to human PTSD. 490 present before trauma exposure may predispose animals to specific stress responses 491 and long-term PTSD-like behaviors. Future studies that focus on the causal relationship 492 between the baseline neural circuit function and behavioral responses during trauma 493 exposure and long-term outcome in PTSD-like behaviors will be needed to understand 494 pathological susceptibility to PTSD. For instance, it will be of particular interest to test 495 whether optogenetic manipulation of specific pathways based on the rsfMRI data during 496 trauma exposure can alter stress response and development of long-term PTSD-like 497 behaviors in animals. 


\section{Methods}

Adult male LE rats (250-350g) were obtained from Charles River Laboratories

501 (Raleigh, North Carolina site). Rats were housed in pairs in Plexiglas cages with

502 controlled ambient temperature $\left(22-24{ }^{\circ} \mathrm{C}\right)$ on a $12 / 12$ hour day-dark schedule. Food and water were provided ad libitum. A total of 87 rats were used in the present study, in which 23 rats were controls and 64 rats were exposed to predator scent using a single-episode predator scent exposure in an inescapable environment paradigm. Two rats were removed from the exposed group before analysis due to health concerns. All rats were used for behavior tests and rsfMRI experiments. All animal procedures were reviewed and approved by the Institutional Animal Care and Use Committee (IACUC) of the Pennsylvania State University. The experimental procedure was summarized in Fig. 1a.

\section{Single-episode predator scent exposure}

Rats were exposed to a single episode of predator scent stress in an inescapable

512 environment for $10 \mathrm{~min}$. The day prior to exposure, each rat was assigned to its own exposure cage (i.e. a Plexiglas cage with no bedding) and habituated to the cage

514 environment for $30 \mathrm{~min}$. The cage was left soiled after habituation. On the exposure day, 515 the rat was placed in its corresponding cage for a total of $12 \mathrm{~min}$. After 2 min of adaptation, 516 a cotton pad sprayed with red fox urine was placed at the right end of the cage for another 51710 mins (Red fox urine, Wild Life Research Center). The pad was put on a wire mesh 518 inside the cage, right below the lid and out of reach of the rat. The control animal was 519 exposed to the same cotton pad without fox urine. During exposure, the rat was recorded 
520 by a video camera. Following predator scent exposure, the rat was returned to his home cage and left undisturbed for 6 days.

522 Elevated plus maze (EPM)

The EPM test was performed to measure the anxiety level 6 days after trauma exposure. The maze consisted of four perpendicular arms forming a cross and elevated $50 \mathrm{~cm}$ from the floor. Two arms were closed, opposed to each other and enclosed by 40$\mathrm{cm}$ high walls. The remaining two arms, also opposed to each other, were completely open. A camera was placed directly above the maze to record the rat movement.

To perform the test, the rat was initially placed in the center of the maze facing one open arm and allowed to freely explore the maze for $5 \mathrm{~min}$. The times spent in the open and closed arms were determined. The ratio of time in open arms versus that in both open

532 level. A lower score of this ratio indicates a higher level of anxiety. The maze was cleaned with a $70 \%$ alcohol solution in water between tests.

\section{CORT Measurement}

Rats $(n=39)$ had blood samples collected to quantify the hormonal response to stress. Corticosterone (CORT) level was measured immediately before predator scent

537 exposure (i.e. $0 \mathrm{~min}$ ) and $30 \mathrm{~min}, 60 \mathrm{~min}$, and $120 \mathrm{~min}$ after exposure to quantify the 538 dynamic response to predator scent stress exposure. Initial blood samples were collected 539 through tail nick, with subsequent time point samples collected by disturbing the tail scab.

540 Samples were collected using heparin coated microtubes with capillary tube inserts (RAM 541 Scientific Safe-T-Fill, Nashville, TN, USA). Samples were centrifuged to obtain plasma 
542 and stored at $-20^{\circ} \mathrm{C}$. The plasma CORT level was then quantified using CORT enzyme-

543 linked immunosorbent assay (ELISA) kit (Enzo life sciences Inc. Ann Arbor, MI. Cat ADI-

544 901-097) according to the manufacturer's manual. The total CORT response for each

545 animal was quantified by the area-under-the-curve of the CORT response curve,

546 calculated by the summation of the CORT amplitude across all time points with the CORT

547 level at 0 min (i.e. baseline) being subtracted at each time point. This measurement took

548 into account both the amplitude and duration of the CORT response and represented the

549 total output of the hypothalamus-pituitary-adrenal axis.

\section{Acclimation and rsfMRI data acquisition}

All rats were scanned before the trauma exposure experiment. Before imaging, protocol was employed to diminish the stress and motion of the subject during scans ${ }^{69}$.

554 The rat was first briefly anesthetized with 2-4\% isoflurane in oxygen (3-5 min). The head was restrained with a bite bar, nose bar and side pads that restricted the head motion. A pair of shoulder bars and a body tube maintained the body in place without impeding ventilation. Animals were allowed to regain consciousness by discontinuing isoflurane

558 after being set up in the restrainer, typically in 10 mins. The rat was then placed in a dark ventilated box and the sound of different MRI sequences was played, presenting an

560 environment mimicking MRI scanning. All rats were given 7 daily acclimation sessions 561 with a gradually increasing acclimation period $(15,30,45,60,60,60$ and 60 min from day 5621 to day 7). A similar approach has also been applied by other research groups ${ }^{70-72}$. 
anesthetized with $2-4 \%$ isoflurane in oxygen and set up in the same restrainer with a builtin birdcage $1 \mathrm{H}$ radiofrequency volume coil. Anesthesia was then discontinued, and the rat was allowed to regain consciousness and placed into the MRI magnet bore. rsfMRI data were obtained using a single shot gradient-echo echo-planar imaging sequence with the parameters of repetition time $(T R)=1 \mathrm{sec}$, echo time $(T E)=13.8 \mathrm{~ms}, 64 \times 64$ matrix size, 20 slices with $1 \mathrm{~mm}$ thickness, field of view $(F O V)=32 \times 32 \mathrm{~mm}^{2}$, in-plane resolution $=500 \times 500 \mu \mathrm{m}^{2}$. Each rat was scanned for $2400 \mathrm{EPI}$ volumes per scan session. Anatomical images were also acquired using a fast spin-echo sequence with the parameters of TR = $1500 \mathrm{~ms} ; \mathrm{TE}=8 \mathrm{~ms} ;$ matrix size $=256 \times 256 ; \mathrm{FOV}=3.2 \times 3.2 \mathrm{~cm}^{2}$; slice number $=20 ;$ slice thickness $=1 \mathrm{~mm} ; \operatorname{RARE}$ factor $=8$.

\section{rsfMRI data processing}

rsfMRI images were preprocessed by performing alignment using Medical Image Visualization and Analysis (MIVA, http://ccni.wpi.edu/), motion correction using SPM 12 (http://www.fil.ion.ucl.ac.uk/spm/), spatial smoothing (Gaussian kernel: FWHM = $0.75 \mathrm{~mm}$ ) and temporal smoothing (band-pass filter, cutoff frequencies: $0.01-0.1 \mathrm{~Hz}$ ), and regression of motion parameters and signals from the white matter and ventricles as described in past studies $20,22,23,73$. Before performing these steps, EPI volumes with relative framewise displacement $(F D)>0.2 \mathrm{~mm}$ and their immediate temporal neighbors were first removed 74. In addition, the first 10 volumes of each rsfMRI run were also removed to ensure the magnetization was at steady state.

Functional connectivity analysis was conducted using region-of-interest (ROI) based correlational analysis. This method evaluated the RSFC by quantifying the Pearson correlation coefficient of regionally averaged spontaneous BOLD signals 
588

589

590

591

592

593

594

595

596

597

598

599

600

601

602

603

604

605

606

607

608

609

610

611

612

613

614

between different brain regions. Seed maps were generated by correlating the regionally averaged seed time course with the time course of each brain voxel. A linear mixed effect model was used to determine the overall effect on RSFC of each group of rats, while removing any random effect from different batches of animals.

\section{References:}

1 Charney, D. S. Psychobiological mechanisms of resilience and vulnerability: implications for successful adaptation to extreme stress. Am J Psychiatry 161, 195216, doi:10.1176/appi.ajp.161.2.195 (2004).

2 Yehuda, R. \& LeDoux, J. Response variation following trauma: a translational neuroscience approach to understanding PTSD. Neuron 56, 19-32, doi:10.1016/j.neuron.2007.09.006 (2007).

3 Feder, A., Nestler, E. J. \& Charney, D. S. Psychobiology and molecular genetics of resilience. Nat Rev Neurosci 10, 446-457, doi:10.1038/nrn2649 (2009).

4 Gilbertson, M. W. et al. Smaller hippocampal volume predicts pathologic vulnerability to psychological trauma. Nat Neurosci 5, 1242-1247, doi:10.1038/nn958 (2002).

5 Bonne, $\mathrm{O}$. et al. Longitudinal MRI study of hippocampal volume in trauma survivors with PTSD. Am J Psychiatry 158, 1248-1251, doi:10.1176/appi.ajp.158.8.1248 (2001).

6 McEwen, B. S., Nasca, C. \& Gray, J. D. Stress Effects on Neuronal Structure: Hippocampus, Amygdala, and Prefrontal Cortex. Neuropsychopharmacology 41, 3-23, doi:10.1038/npp.2015.171 (2016).

7 Logue, M. W. et al. Smaller Hippocampal Volume in Posttraumatic Stress Disorder: A Multisite ENIGMA-PGC Study: Subcortical Volumetry Results From Posttraumatic Stress Disorder Consortia. Biol Psychiatry 83, 244-253, doi:10.1016/j.biopsych.2017.09.006 (2018). 
6158 Yehuda, R. Advances in understanding neuroendocrine alterations in PTSD and their therapeutic implications. Ann N Y Acad Sci 1071, 137-166, doi:10.1196/annals.1364.012 (2006). Milad, M. R. et al. Neurobiological basis of failure to recall extinction memory in posttraumatic stress disorder. Biol Psychiatry 66, 1075-1082, doi:10.1016/j.biopsych.2009.06.026S0006-3223(09)00896-8 [pii] (2009). cognitive interference: a monozygotic twin study of posttraumatic stress disorder. Am J Psychiatry 168, 979-985, doi:10.1176/appi.ajp.2011.09121812 (2011). post-traumatic stress disorder: face validity. Frontiers in neuroscience 7, 89, doi:10.3389/fnins.2013.00089 (2013).

12 Deslauriers, J., Toth, M., Der-Avakian, A. \& Risbrough, V. B. Current Status of Animal Models of Posttraumatic Stress Disorder: Behavioral and Biological Phenotypes, and Future Challenges in Improving Translation. Biol Psychiatry 83,

14 Cohen, H. \& Zohar, J. An animal model of posttraumatic stress disorder: the use of cut-off behavioral criteria. Ann N Y Acad Sci 1032, 167-178 (2004). 895-907, doi:10.1016/j.biopsych.2017.11.019 (2018).

15 Roelofs, K. Freeze for action: neurobiological mechanisms in animal and human

63816 Fanselow, M. S. Neural organization of the defensive behavior system responsible 639 freezing. Philos Trans $R$ Soc Lond B Biol Sci 372, doi:10.1098/rstb.2016.0206 (2017). for fear. Psychon Bull Rev 1, 429-438, doi:10.3758/BF03210947 (1994).

17 Liang, Z., King, J. \& Zhang, N. Uncovering intrinsic connectional architecture of functional networks in awake rat brain. J Neurosci 31, 3776-3783 (2011).

8 Liang, Z., King, J. \& Zhang, N. Anticorrelated resting-state functional connectivity in awake rat brain. Neuroimage 59, 1190-1199, doi:S1053-8119(11)00896-2 [pii]10.1016/j.neuroimage.2011.08.009 (2012). 
64519 Ma, Z. et al. Functional atlas of the awake rat brain: A neuroimaging study of rat 646 brain specialization and integration. Neuroimage 170, 95-112, doi:10.1016/j.neuroimage.2016.07.007 (2018).

64820 Ma, Z. \& Zhang, N. Temporal transitions of spontaneous brain activity. eLife 7, 649 doi:10.7554/eLife.33562 (2018).

651

21 Gao, Y. R. et al. Time to wake up: Studying neurovascular coupling and brain-wide circuit function in the un-anesthetized animal. Neuroimage, doi:10.1016/j.neuroimage.2016.11.069 (2016).

654

23 Liang, Z., King, J. \& Zhang, N. Neuroplasticity to a single-episode traumatic stress revealed by resting-state fMRI in awake rats. Neuroimage 103, 485-491, doi:10.1016/j.neuroimage.2014.08.050 (2014).

24 Ubeda-Contreras, J., Marin-Blasco, I., Nadal, R. \& Armario, A. Brain c-fos 659 expression patterns induced by emotional stressors differing in nature and intensity. Brain structure \& function 223, 2213-2227, doi:10.1007/s00429-018-1624-2 (2018).

66125 Janitzky, K., D'Hanis, W., Krober, A. \& Schwegler, H. TMT predator odor activated 662 neural circuit in C57BL/6J mice indicates TMT-stress as a suitable model for correlates of sustained fear in the anterolateral part of the bed nucleus of stria terminalis. Neurobiology of learning and memory 131, 137-146, doi:10.1016/j.nlm.2016.03.020 (2016).

66927 Gungor, N. Z. \& Pare, D. CGRP inhibits neurons of the bed nucleus of the stria 670 terminalis: implications for the regulation of fear and anxiety. J Neurosci 34, 60-65, doi:10.1523/JNEUROSCI.3473-13.2014 (2014).

67228 Ramirez, F., Moscarello, J. M., LeDoux, J. E. \& Sears, R. M. Active avoidance requires a serial basal amygdala to nucleus accumbens shell circuit. $J$ Neurosci 35, 3470-3477, doi:10.1523/JNEUROSCI.1331-14.2015 (2015). 
67529 Da Cunha, C., Gomez, A. A. \& Blaha, C. D. The role of the basal ganglia in 676 motivated behavior. Rev Neurosci 23, 747-767, doi:10.1515/revneuro-2012-0063

12)

67830 Mitrofanis, J. Some certainty for the "zone of uncertainty"? Exploring the function of the zona incerta. Neuroscience

130 , $1-15$ doi:10.1016/j.neuroscience.2004.08.017 (2005).

31 Hunt, A. J., Jr. et al. Paraventricular hypothalamic and amygdalar CRF neurons synapse in the external globus pallidus. Brain structure \& function 223, 2685-2698, doi:10.1007/s00429-018-1652-y (2018).

32 Dopfel, D. \& Zhang, N. Mapping stress networks using functional magnetic resonance imaging in awake animals. Neurobiol Stress 9, 251-263, doi:10.1016/j.ynstr.2018.06.002 (2018).

33 Paré, D. \& Quirk, G. J. When scientific paradigms lead to tunnel vision: lessons from the study of fear. npj Science of Learning 2, 6, doi:10.1038/s41539-017-00074 (2017).

35 Holbrook, T. L., Hoyt, D. B., Stein, M. B. \& Sieber, W. J. Perceived threat to life

34 Gruene, T. M., Flick, K., Stefano, A., Shea, S. D. \& Shansky, R. M. Sexually divergent expression of active and passive conditioned fear responses in rats. predicts posttraumatic stress disorder after major trauma: risk factors and functional outcome. J Trauma 51, 287-292; discussion 292-283 (2001).

38 Rao, R. P., Anilkumar, S., McEwen, B. S. \& Chattarji, S. Glucocorticoids protect against the delayed behavioral and cellular effects of acute stress on the amygdala. 
70640 Whitaker, A. M. \& Gilpin, N. W. Blunted hypothalamo-pituitary adrenal axis

707

708

709

710

711

712

713

714

715

716

717

718

719

720

721

722

723

724

725

726

727

728

729

730

731

732

733

734

735 response to predator odor predicts high stress reactivity. Physiol Behav 147, 1622, doi:10.1016/j.physbeh.2015.03.033 (2015).

41 Yehuda, R., McFarlane, A. C. \& Shalev, A. Y. Predicting the development of posttraumatic stress disorder from the acute response to a traumatic event. Biol Psychiatry 44, 1305-1313 (1998).

42 Delahanty, D. L., Raimonde, A. J. \& Spoonster, E. Initial posttraumatic urinary cortisol levels predict subsequent PTSD symptoms in motor vehicle accident victims. Biol Psychiatry 48, 940-947 (2000).

43 Weaver, I. C. et al. Reversal of maternal programming of stress responses in adult offspring through methyl supplementation: altering epigenetic marking later in life. J Neurosci 25, 11045-11054, doi:10.1523/JNEUROSCI.3652-05.2005 (2005).

44 Schelling, G. et al. Efficacy of hydrocortisone in preventing posttraumatic stress disorder following critical illness and major surgery. Ann N Y Acad Sci 1071, 4653, doi:10.1196/annals.1364.005 (2006).

45 Zohar, J. et al. High dose hydrocortisone immediately after trauma may alter the trajectory of PTSD: interplay between clinical and animal studies. European neuropsychopharmacology : the journal of the European College of Neuropsychopharmacology 21, 796-809, doi:10.1016/j.euroneuro.2011.06.001 (2011).

46 Cullinan, W. E., Herman, J. P., Battaglia, D. F., Akil, H. \& Watson, S. J. Pattern and time course of immediate early gene expression in rat brain following acute stress. Neuroscience 64, 477-505 (1995).

47 Dielenberg, R. A., Hunt, G. E. \& McGregor, I. S. "When a rat smells a cat": the distribution of Fos immunoreactivity in rat brain following exposure to a predatory odor. Neuroscience 104, 1085-1097 (2001).

48 Friedrich, R. W. Olfactory neuroscience: beyond the bulb. Curr Biol 21, R438-440, doi:10.1016/j.cub.2011.04.036 (2011).

49 Ferrer, N. G. Efferent projections of the anterior olfactory nucleus. J Comp Neurol 137, 309-320, doi:10.1002/cne.901370305 (1969). 
73650 Gozzi, A. et al. A neural switch for active and passive fear. Neuron 67, 656-666, doi:10.1016/j.neuron.2010.07.008 (2010).

739

740

51 Kondoh, K. et al. A specific area of olfactory cortex involved in stress hormone responses to predator odours. Nature 532, 103-106, doi:10.1038/nature17156 (2016). sustained fear: a tribute to Dr. Lennart Heimer. Brain structure \& function 213, 2942, doi:10.1007/s00429-008-0183-3 (2008).

54 Somerville, L. H., Whalen, P. J. \& Kelley, W. M. Human bed nucleus of the stria terminalis indexes hypervigilant threat monitoring. Biol Psychiatry 68, 416-424, doi:10.1016/j.biopsych.2010.04.002 (2010).

55 Grupe, D. W. \& Nitschke, J. B. Uncertainty and anticipation in anxiety: an 750 integrated neurobiological and psychological perspective. Nat Rev Neurosci 14, 488-501, doi:10.1038/nrn3524 (2013).

752

56 Davis, M. Neural systems involved in fear and anxiety measured with fear753

754 potentiated startle. Am Psychol 61, 741-756, doi:10.1037/0003-066X.61.8.741 (2006).

756

58 Dong, H. W. \& Swanson, L. W. Organization of axonal projections from the anterolateral area of the bed nuclei of the stria terminalis. J Comp Neurol 468, 277298, doi:10.1002/cne.10949 (2004).

59 Tovote, P., Fadok, J. P. \& Luthi, A. Neuronal circuits for fear and anxiety. Nat Rev Neurosci 16, 317-331, doi:10.1038/nrn3945 (2015).

60 Sierra-Mercado, D., Padilla-Coreano, N. \& Quirk, G. J. Dissociable roles of prelimbic and infralimbic cortices, ventral hippocampus, and basolateral amygdala 
in the expression and extinction of conditioned fear. Neuropsychopharmacology 36, 529-538, doi:10.1038/npp.2010.184 (2011).

61 Diehl, M. M. et al. Active avoidance requires inhibitory signaling in the rodent prelimbic prefrontal cortex. eLife 7, doi:10.7554/eLife.34657 (2018).

62 Vertes, R. P. \& Kocsis, B. Brainstem-diencephalo-septohippocampal systems controlling the theta rhythm of the hippocampus. Neuroscience 81, 893-926 (1997).

63 Buzsaki, G. Theta oscillations in the hippocampus. Neuron 33, 325-340 (2002).

64 Lesting, J. et al. Directional theta coherence in prefrontal cortical to amygdalohippocampal pathways signals fear extinction. PLoS One 8, e77707, doi:10.1371/journal.pone.0077707 (2013).

65 Marek, R. et al. Hippocampus-driven feed-forward inhibition of the prefrontal cortex mediates relapse of extinguished fear. Nat Neurosci 21, 384-392, doi:10.1038/s41593-018-0073-9 (2018).

66 Zhang, J. et al. Decreased gray matter volume in the left hippocampus and bilateral calcarine cortex in coal mine flood disaster survivors with recent onset PTSD. Psychiatry Res 192, 84-90, doi:10.1016/j.pscychresns.2010.09.001 (2011).

67 Lebow, M. A. \& Chen, A. Overshadowed by the amygdala: the bed nucleus of the stria terminalis emerges as key to psychiatric disorders. Molecular psychiatry 21, 450-463, doi:10.1038/mp.2016.1 (2016).

68 Schelling, G., Roozendaal, B. \& De Quervain, D. J. Can posttraumatic stress disorder be prevented with glucocorticoids? Ann N Y Acad Sci 1032, 158-166, doi:10.1196/annals.1314.013 (2004).

69 King, J. A. et al. Procedure for minimizing stress for fMRI studies in conscious rats. J Neurosci Methods 148, 154-160 (2005).

70 Bergmann, E., Zur, G., Bershadsky, G. \& Kahn, I. The Organization of Mouse and Human Cortico-Hippocampal Networks Estimated by Intrinsic Functional Connectivity. Cereb Cortex 26, 4497-4512, doi:10.1093/cercor/bhw327 (2016).

71 Yoshida, K. et al. Physiological effects of a habituation procedure for functional MRI in awake mice using a cryogenic radiofrequency probe. J Neurosci Methods 274, 38-48, doi:10.1016/j.jneumeth.2016.09.013 (2016). 
79672 Chang, P. C. et al. Novel method for functional brain imaging in awake minimally 797 restrained rats. J Neurophysiol 116, 61-80, doi:10.1152/jn.01078.2015 (2016).

79873 Liang, Z., Liu, X. \& Zhang, N. Dynamic resting state functional connectivity in 799 awake and anesthetized rodents. Neuroimage 104, 89-99, 800 doi:10.1016/j.neuroimage.2014.10.013 (2015).

80174 Power, J. D., Barnes, K. A., Snyder, A. Z., Schlaggar, B. L. \& Petersen, S. E. 802 Spurious but systematic correlations in functional connectivity MRI networks arise 803 from subject motion. Neuroimage 59, 2142-2154, 804 doi:10.1016/j.neuroimage.2011.10.018 (2012).

805

806

807 
(a) Experimental schedule diagram

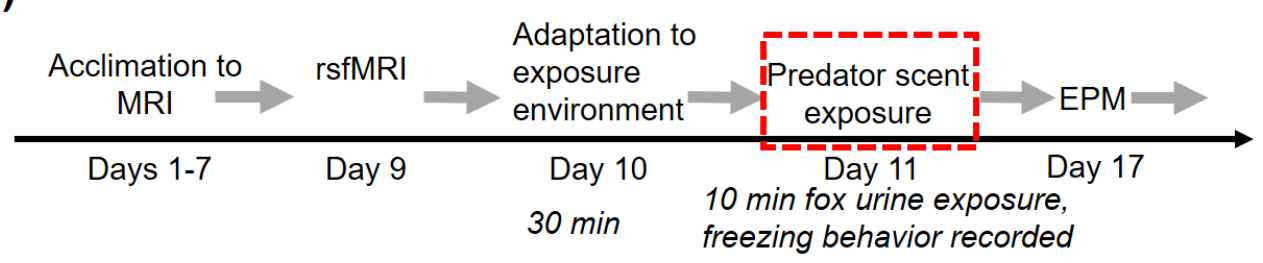

(b)
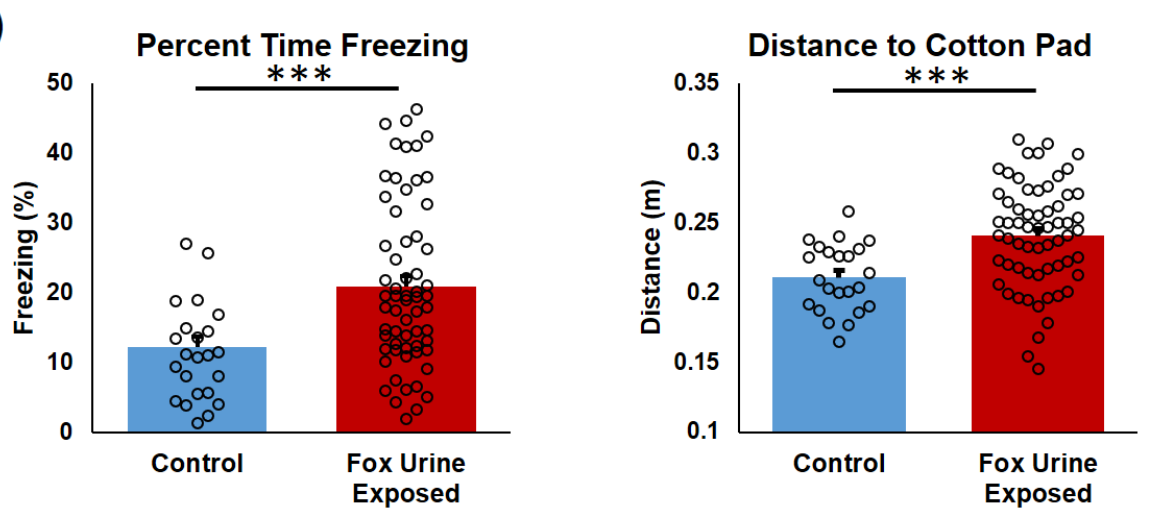

(c)
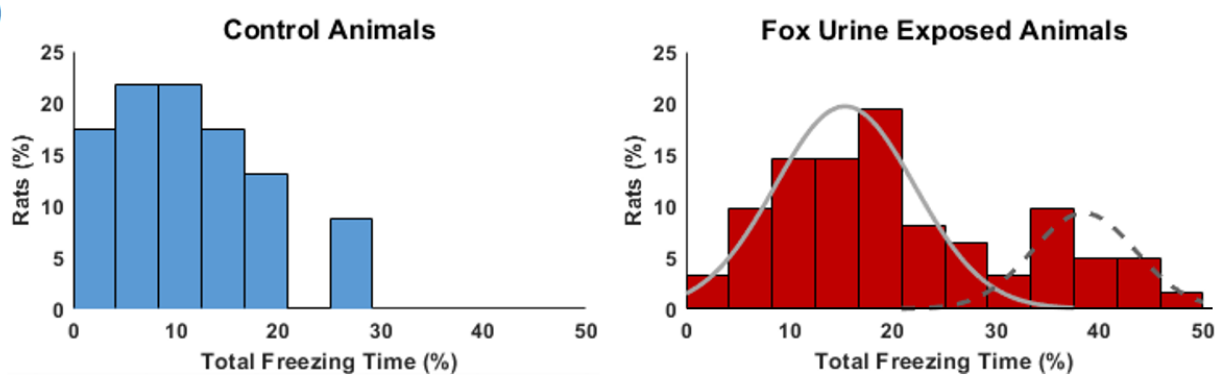

(d)

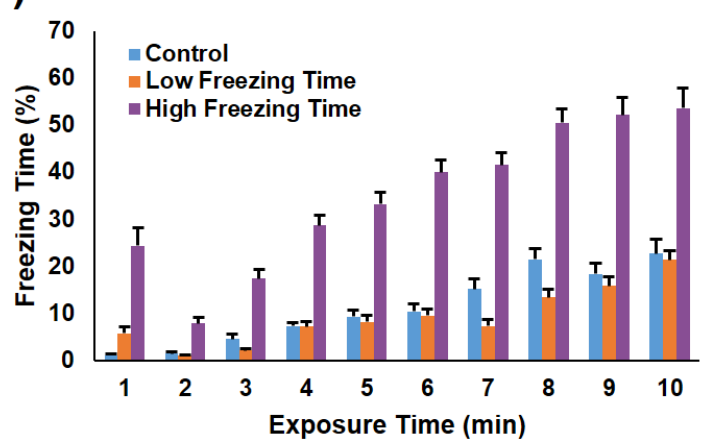

809 Figure 1. Freezing time during the 10-min exposure session in control $(n=23)$ and fox urine exposed ( $n=62$ ) groups. a) Diagram of experimental schedule. b) Fox urine exposed animals showed a significantly longer freezing time (left, $p<0.0005$, in percentage) and greater mean distance (right, $p<0.0007$, in meters) from the cotton pad than controls. c) The distributions of individual animals' freezing time in the control group (left) and the exposed group (right) displayed as probability histograms. The freezing time distribution in exposed animals was fit to a Gaussian mixture model which demonstrated a bimodal distribution. d) Freezing time each min plotted as a function of time in lowfreezing $(n=21)$, high-freezing $(n=21)$ and control animals during fox urine exposure. The high freezing animals displayed higher freezing phenotype during the first minute of 
819 exposure, with a significantly longer freezing time than both low freezing and control 820 animals from the 1 st minute through the 10th minute $(p<0.005$ for each minute of 821 exposure).

822 
(a)

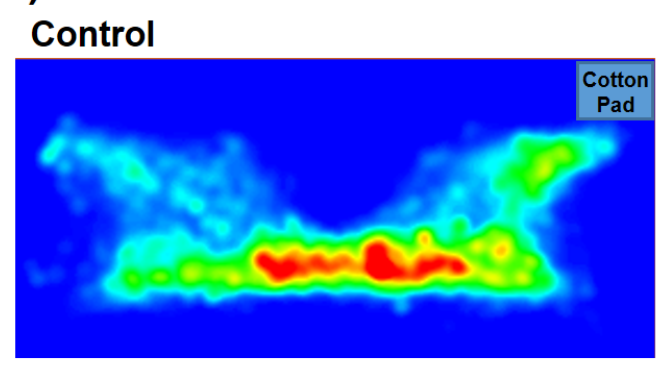

Time spent:

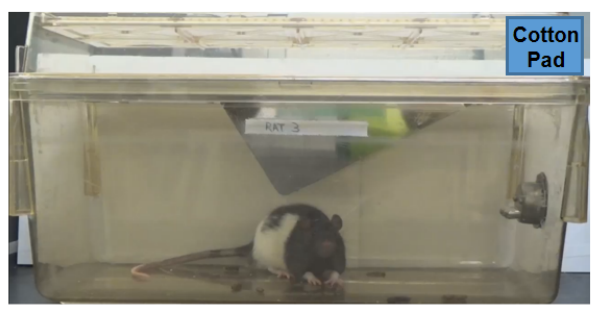

(b)

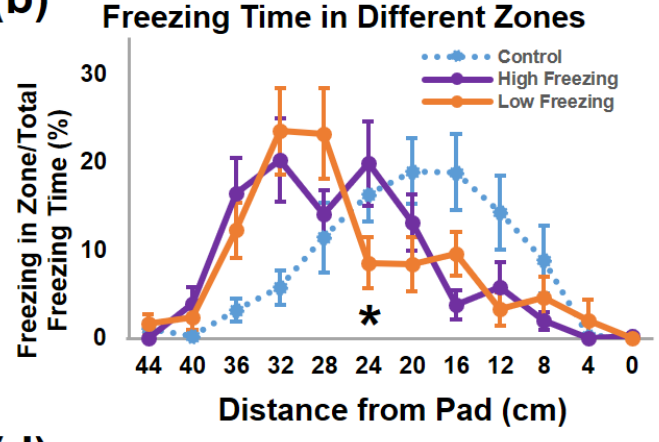

(d)

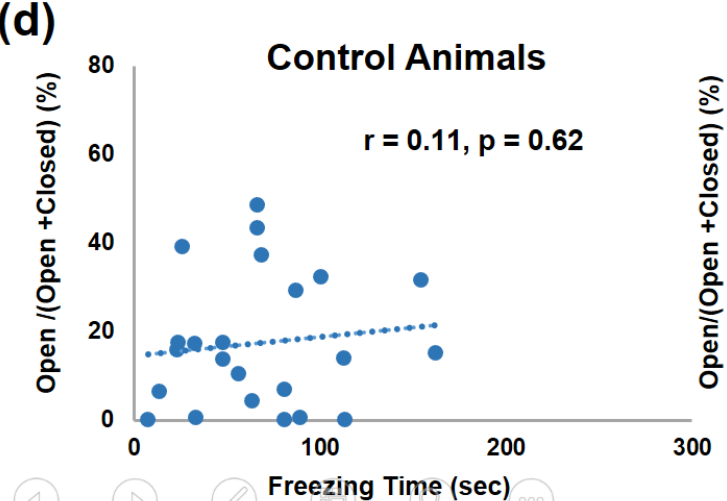

Low freezing animals

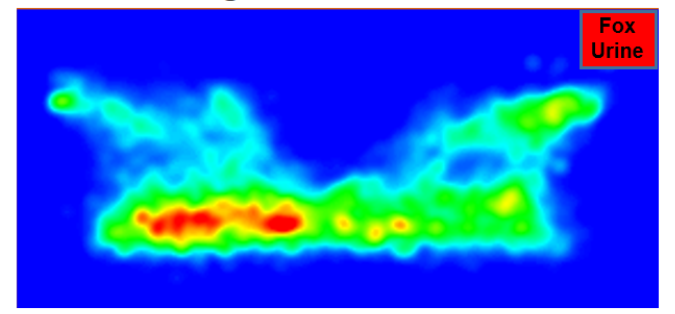

High freezing animals

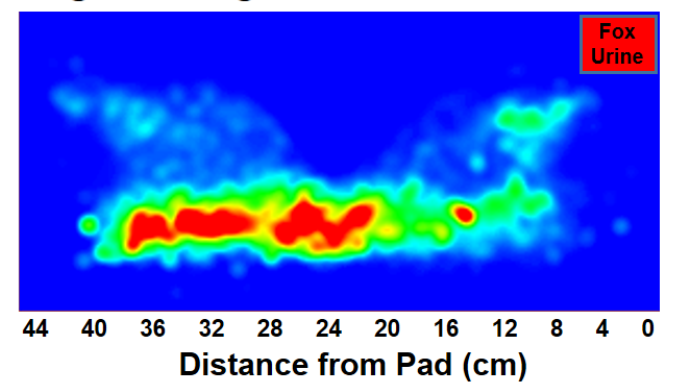

(c)
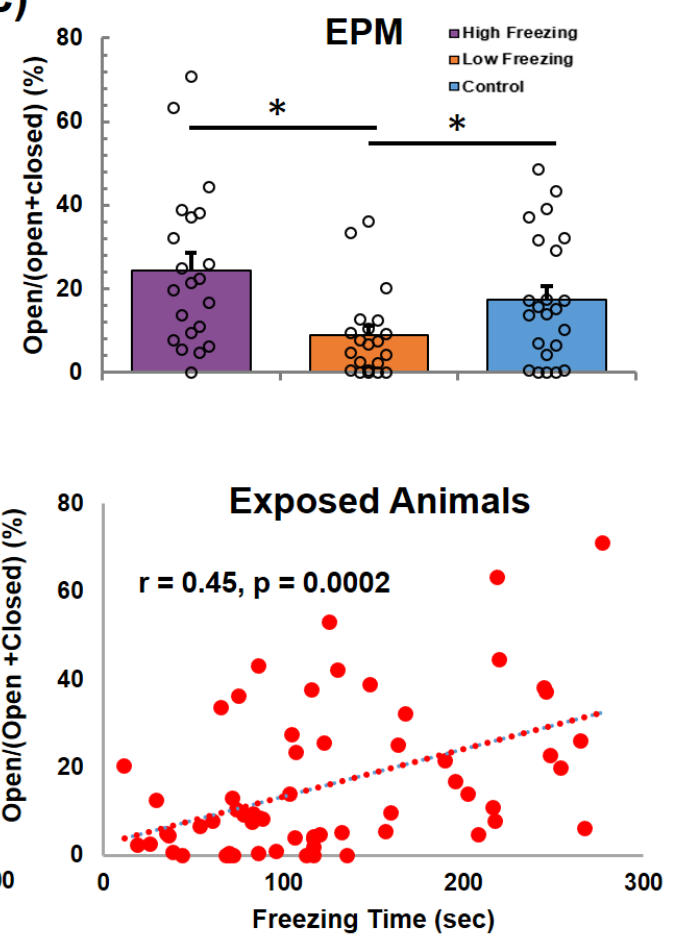

Figure 2. Low-freezing animals exhibited stronger stress response during fox urine exposure and higher anxiety 6 days after exposure. a) Heat maps of the total time at each location for the control, low freezing, and high freezing groups. The control group showed no evident bias for either side of the cage. The low freezing group showed a strong bias toward the far end (from the pad) of the cage, while the high freezing rats also showed a bias toward the far end of the cage, but to a lesser extent. b) The distribution of the percent 830 freezing time for each group across the cage, divided into 12 equivalent-sized zones. The $831 \mathrm{x}$-axis indicates the distance to the pad from the center of each zone. Similar to the 
832 distributions of total time spent, the percent freezing time in control rats displayed little 833 bias for either side of the cage. High and low freezing animals showed a bias of percent 834 freezing time toward the far end of the cage, with the low-freezing rats showing even 835 stronger bias than high-freezing animals, evidenced by significantly lower percent 836 freezing time of the low-freezing group in the zone $24 \mathrm{~cm}$ away from the pad compared 837 to the high-freezing group ( $p=0.026)$. c) Elevated plus maze performed 6 days post 838 exposure in all three groups of animals. Low-freezing animals showed a significantly 839 lower EPM score (open/open+closed arm time) compared to both control $(p=0.030)$ and 840 high-freezing animals ( $p=0.0017$ ), while no significant difference was seen between 841 control and high freezing groups. d) The freezing time was positively correlated to the 842 EPM score across all exposed animals $(r=0.45, p=0.0002)$, but not across control 843 animals $(r=0.11, p=0.62)$.

844 
(a)

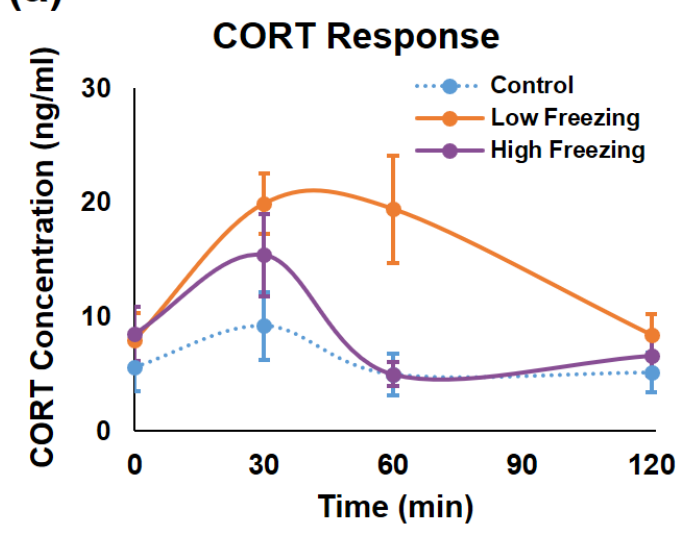

(c)

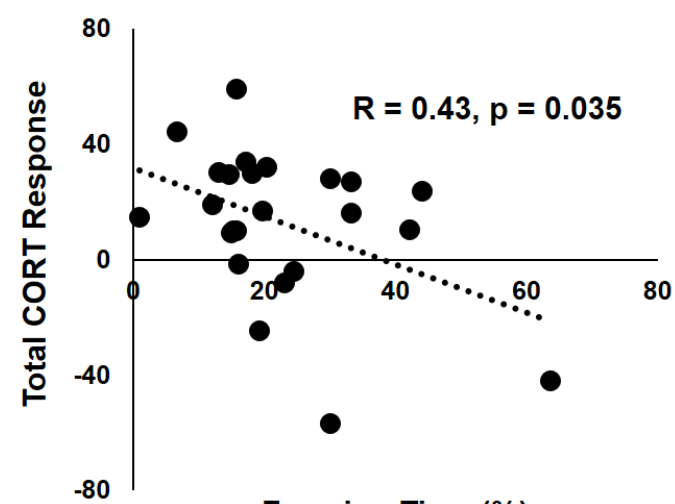

Freezing Time (\%) (b)

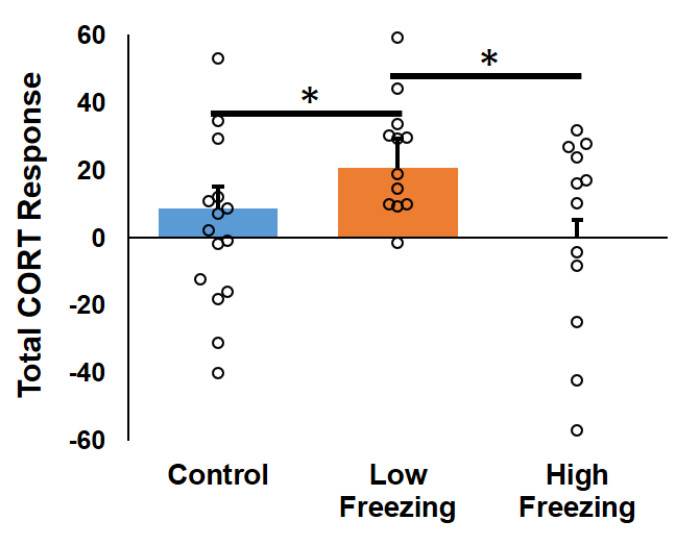

(d)

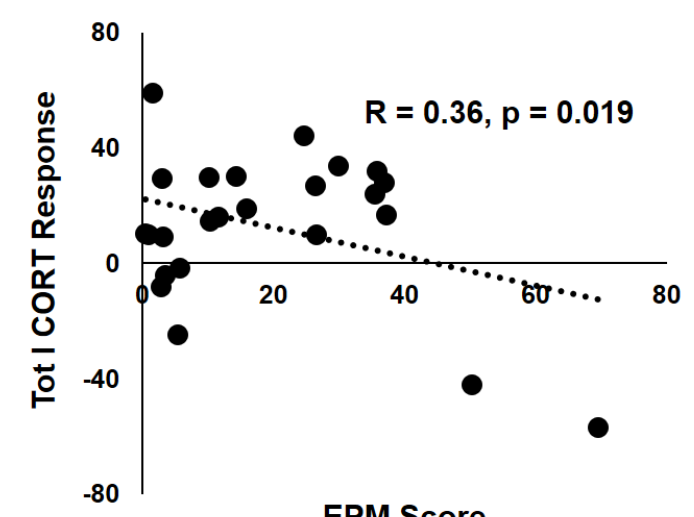

846

847

848

Figure 3. Corticosterone (CORT) response to fox urine exposure. a) The time course of CORT (in $\mathrm{ng} / \mathrm{ml}$ ) for high-, low-freezing and control animals. There was a significant difference in CORT level changes over time among three groups (Two-way repeated ANOVA, $\left.F_{\text {group } x \text { time }}(6,108)=2.84, p=0.023\right)$. Control animals showed no appreciable CORT change across time (One-way repeated ANOVA, $F_{\text {time }}(3,56)=0.8, p=0.50$ ). Highfreezing animals exhibited a relatively short CORT response, peaking at $30 \mathrm{~min}$ and returning to baseline at $60 \mathrm{~min}$ (Tukey-Kramer test, $30 \mathrm{~min}$ vs $60 \mathrm{~min}: p=0.049 ; 0 \mathrm{~min}$ vs 60 min: $p=0.54 ; 60$ min vs $120 \mathrm{~min}: p=0.93)$. The peak amplitude of CORT response at $30 \mathrm{~min}$ was comparable between high- and low-freezing animals (Tukey-Kramer test, $p=0.59$ ). However, relative to high-freezing rats, the CORT response in low-freezing animals was prolonged, maintained at a high level at $60 \mathrm{~min}$ (Tukey-Kramer test, $30 \mathrm{~min}$ vs 60 min: $p=1 ; 0$ min vs 60 min: $p=0.0005$ ) and had a delayed return to baseline at $120 \mathrm{~min}$ (Tukey-Kramer test, $60 \mathrm{~min}$ vs $120 \mathrm{~min}: \mathrm{p}=0.0098$; 0 min vs $120 \mathrm{~min}: \mathrm{p}=1$ ). In addition, low freezers' CORT level was significantly higher at 60 min than high freezers (Tukey-Kramer test, $p=0.0039$ ). b) Total CORT response was quantified by the areaunder-the-curve (AUC) of the CORT response curve, with the baseline CORT level (i.e. $0 \mathrm{~min}$ ) being subtracted from each time point. This quantification method takes into account both the amplitude and length of the hormone response and represents the total output of the hypothalamus-pituitary-adrenal axis during exposure. Low-freezing animals showed a significant higher total CORT response than high-freezing $(p=0.031)$ and control $(p=0.017)$ animals. $c)$ Significant correlation between the total CORT responsive 
867 (y-axis) and freezing time during exposure across all fox urine exposed animals $(r=0.43$, $868 p=0.035)$. d) Significant correlation $(r=0.36, p=0.019)$ between the total CORT 869 response (y-axis) and EPM score (open/open+closed arm time, $x$-axis) measured 6 days 870 post exposure across all animals tested.

871 
(a)
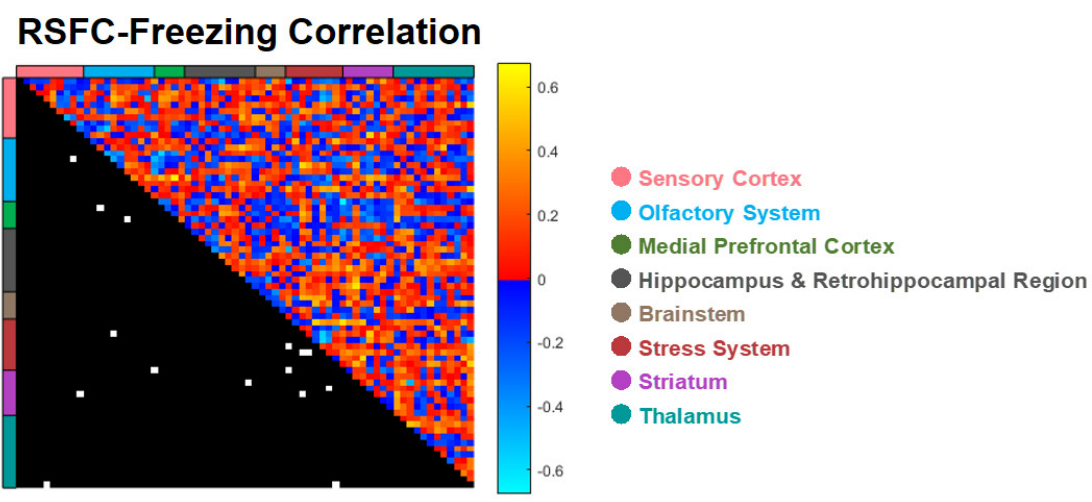

(b)
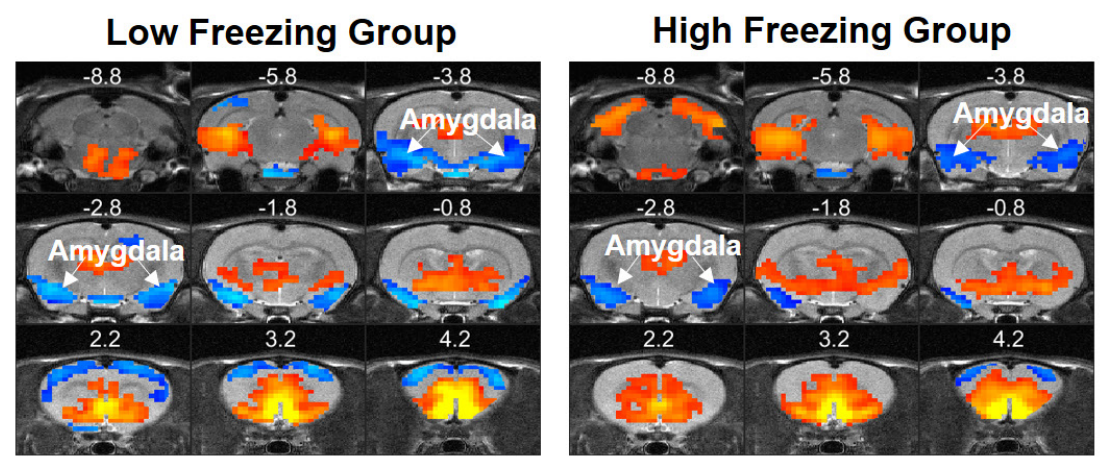

\section{AON}

ACC
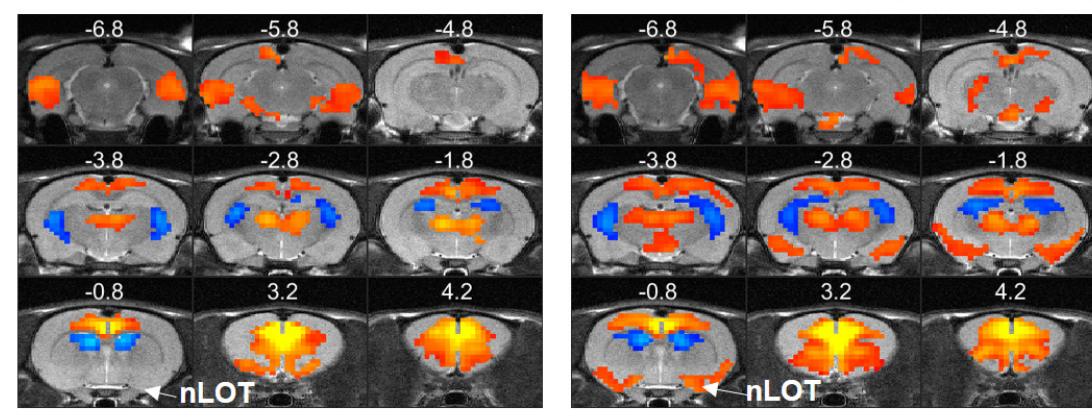

CC
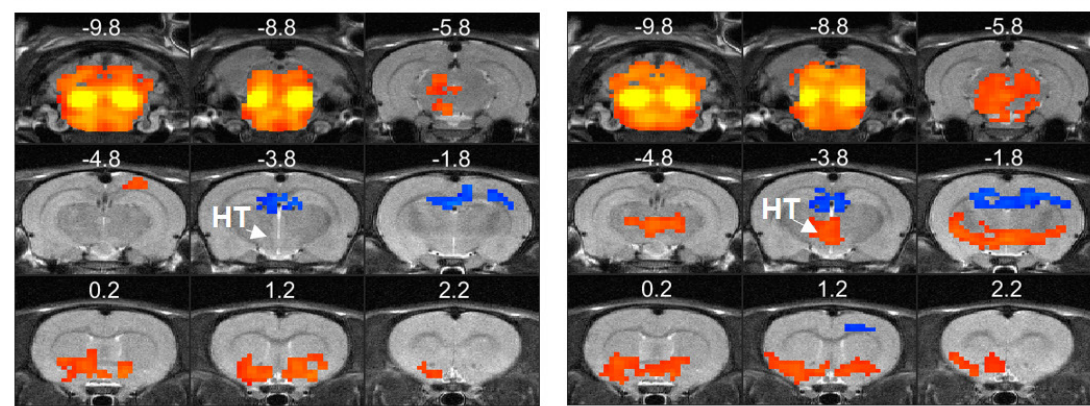

Figure 4. a) Correlations between pre-trauma RSFC and freezing time for all connections (upper triangle) and super-threshold connections (lower triangle, each white element represents a super-threshold connection). Brain regions are arranged based on the brain system (color coded) they belong to. b) Three representative RSFC maps, generated with ACC, AON and PBn as the seed, respectively (colored voxels, $p<0.05$, FDR corrected).

878 Distance to Bregma was labeled at the top of each slice. ACC, anterior cingulate cortex; 879 AON, anterior olfactory nucleus; HT, hypothalamus; nLOT, lateral olfactory tract nucleus; 880 PBn, parabrachial nucleus. 


\section{Pre-existing neural circuits associated with freezing}

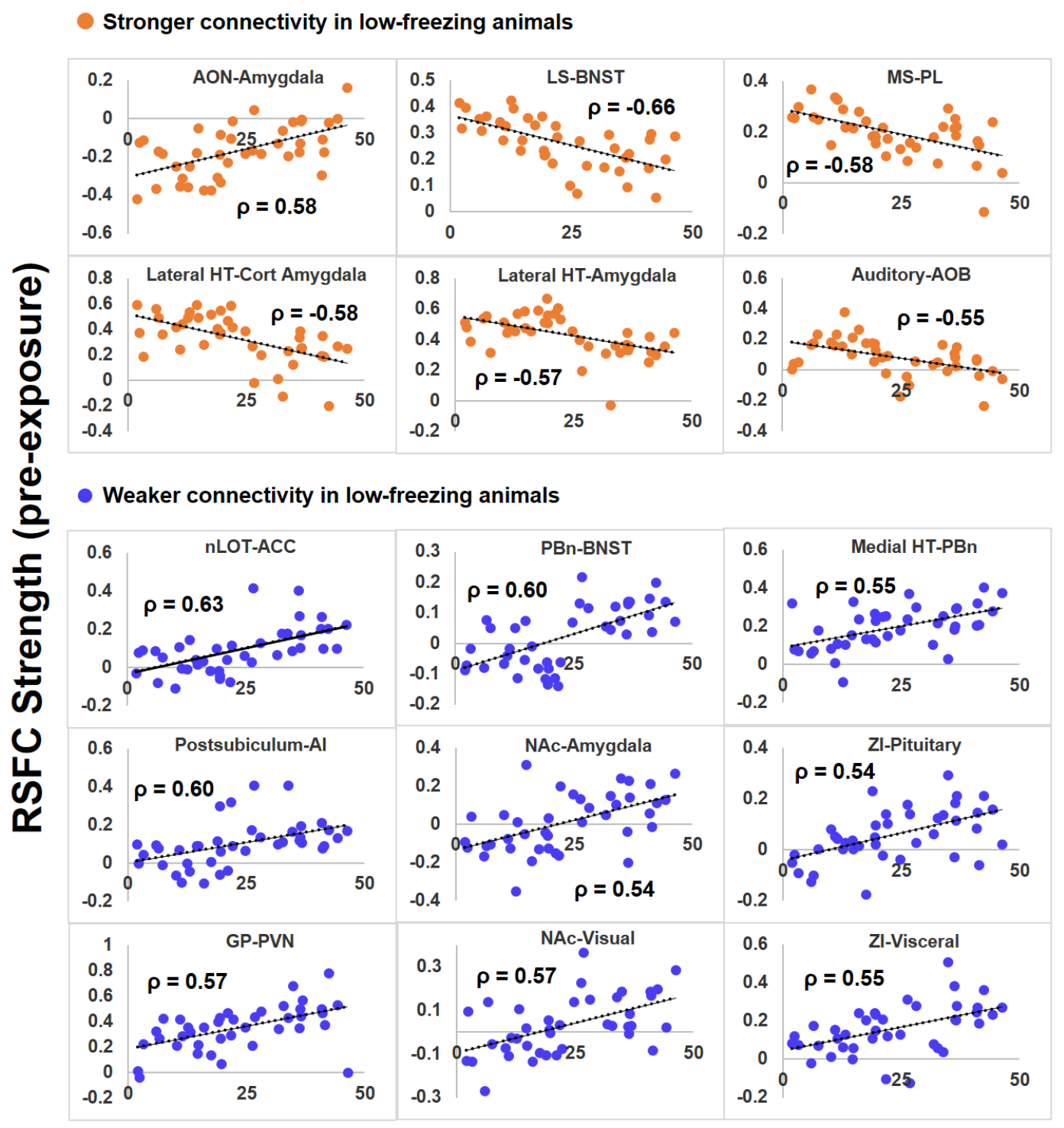

\section{Freezing Time (\%)}

Figure 5. Neural circuits with pre-trauma resting-state functional connectivity (y-axis) correlated $(p<0.05$, FDR corrected) with percentage freezing time ( $x$-axis) in all fox urine exposed animals ( $n=62)$. AON, anterior olfactory nucleus; LS, lateral septum; BNST, bed nucleus of the stria terminalis; MS, medial septum; PL, prelimbic cortex; HT, hypothalamus; Cort Amygdala, cortical amygdala; AOB, accessory olfactory Bulb; nLOT, lateral olfactory tract nucleus; $\mathrm{ACC}$, anterior cingulate cortex; $\mathrm{PBn}$, parabrachial nucleus; Al, postsubiculum-agranular insula; NAc, nucleus accumbens; ZI, zona inserta; GP, globus pallidus; PVN, paraventricular nucleus. 


\section{Diagram of freezing-associated network}
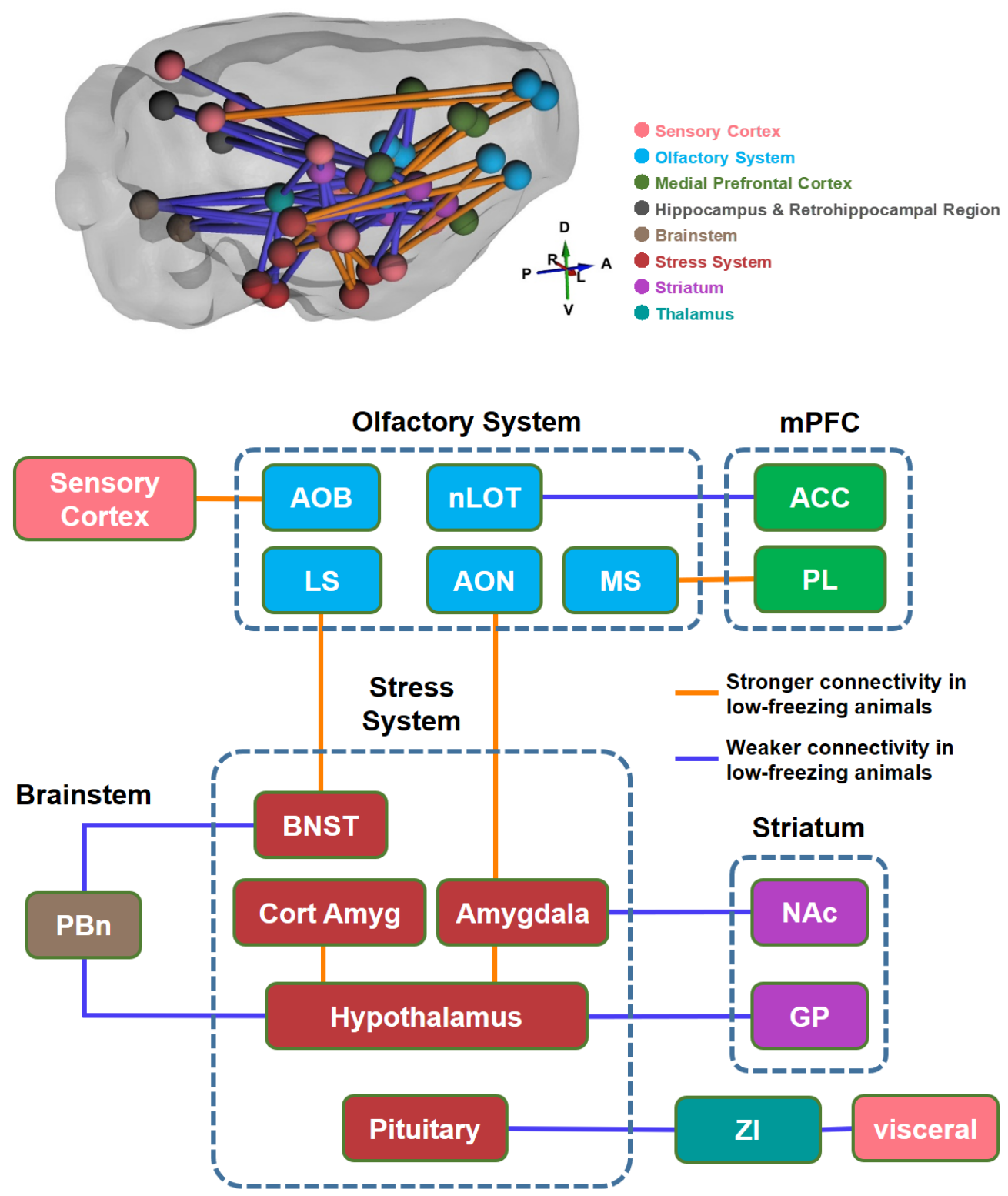

893 Figure 6. Diagram of freezing-associated neural network, including neural circuits 894 showing significant correlation between pre-trauma RSFC and total freezing time shown 895 in Fig. 5. Brain regions are grouped and color coded based on the brain systems they 896 belong to. Orange lines indicate stronger connectivity in low-freezing animals. Blue lines 898 indicate weaker connectivity in low-freezing animals. See the caption of Fig. 5 for the abbreviation. 